\title{
Design of Festival Sentiment Classifier Based on Social Network
}

\author{
Huilin Yuan $\mathbb{D}^{1,2}$ Yufan Song $\mathbb{D}^{2},{ }^{2}$ Jianlu $\mathrm{Hu}^{2}$ and Yatao $\mathrm{Ma}^{3}$ \\ ${ }^{1}$ College of Management, Northeastern University at Qinhuangdao, Qinhuangdao 066004, China \\ ${ }^{2}$ College of Information Science and Engineering, Northeastern University, Shenyang 110819, China \\ ${ }^{3}$ College of Computer and Communication Engineering, Northeastern University, Shenyang 110819, China \\ Correspondence should be addressed to Huilin Yuan; yhlneuq@163.com and Yufan Song; songyufan_vip@126.com
}

Received 6 April 2020; Revised 4 July 2020; Accepted 15 July 2020; Published 7 August 2020

Academic Editor: Ezequiel López-Rubio

Copyright $\odot 2020$ Huilin Yuan et al. This is an open access article distributed under the Creative Commons Attribution License, which permits unrestricted use, distribution, and reproduction in any medium, provided the original work is properly cited.

\begin{abstract}
With the development of society, more and more attention has been paid to cultural festivals. In addition to the government's emphasis, the increasing consumption in festivals also proves that cultural festivals are playing increasingly important role in public life. Therefore, it is very vital to grasp the public festival sentiment. Text sentiment analysis is an important research content in the field of machine learning in recent years. However, at present, there are few studies on festival sentiment, and sentiment classifiers are also limited by domain or language. The Chinese text classifier is much less than the English version. This paper takes Sina Weibo as the text information carrier and Chinese festival microblogs as the research object. CHN-EDA is used to do Chinese text data augmentation, and then the traditional classifiers $\mathrm{CNN}, \mathrm{DNN}$, and naïve Bayes are compared to obtain a higher accuracy. The matching optimizer is selected, and relevant parameters are determined through experiments. This paper solves the problem of unbalanced Chinese sentiment data and establishes a more targeted festival text classifier. This festival sentiment classifier can collect public festival emotion effectively, which is beneficial for cultural inheritance and business decisions adjustment.
\end{abstract}

\section{Introduction}

In recent years, with the continuous improvement of people's living standards and the rapid development of e-commerce technology, both the growing consumption tendency in festivals and the relevant festival policies promulgated by the states show that cultural festivals are increasingly valued. Abroad, Americans spend more than 1 trillion dollars on Christmas day and English spend more than 2 billion pounds according to report from MuchNeeded. In China, Chia et al. [1] analysed the excess returns phenomenon in the Hong Kong stock market due to the preand post-Chinese New Year holiday effect. That investors were affected by the holiday effect in stock trading was explained with finance knowledge. Yuan and Gupta [2] studied the impact of the Chinese Lunar New Year on stock markets in six Asian countries and regions, including China, Hong Kong, and Japan, and found positive results brought by preholiday effect. According to the Consumption Trend Report on Traditional Festivals released by Freshippo, the sale growth of the four traditional festivals-Spring Festival,
Tomb-Sweeping Day, Dragon Boat Festival, and Mid-Autumn Festival-is far higher than that of foreign festivals in China, with a sale increase of more than $20 \%$ in 2019. Chinese government has also published the opinions on the implementation of the inheritance and development project of Chinese traditional culture in 2017. All the facts have proved that the festivals affect peoples' lives in almost every aspect. Therefore, if the public festival emotions can be grasped correctly, reasonable emotional analysis is conducive to a better cultural development and economic decisions.

Nowadays, as a tool for communication and entertainment, social platforms are frequently used. For example, Facebook, a large and well-known social network, has about 215 million unique visitors in the United States and more than 2 billion users. Twitter reported 126 million daily users in 2018. Due to the ample functions of social media such as retweets, comments, and thumbs up, it is more possible for an individual to be influenced by others and show their thoughts, viewpoints, and attitudes on the social platform $[3,4]$. Hence, these social texts gradually become suitable 
resource for data mining and text analysis. Many valuable studies about sentiment analysis and prediction are done based on data mined from social media [5-9].

Text mining by using social networks data has been studied for several years. Researchers studied on identity [10], lexicon [11,12], and social relationship [13] based on social network information. Some customer opinions [14] and other short texts $[15,16]$ on social software are often used as information sources to study emotion classification. However, due to the limitations of language structure and data type, Chinese text classifiers are less studied than the English version, and the accuracy of classifiers in some specific fields cannot be guaranteed. Through the processing of data, the comparison of classifiers, and the determination of parameters, a Chinese festival sentiment classifier with good prediction accuracy can be established in this paper. In the context of the current worldwide, a better understanding of the public's emotional tendency and the development trend of public opinion on traditional festivals is beneficial for the country to let the public better inherit and carry forward the traditional culture of the nation. For businesses, it is helpful to make the right decision, so as to improve their own greater benefits with less cost.

This paper is organized as follows. Related work is described in Section 2. And, then the methodology, including CHN-EDA, CNN, DNN, and naïve Bayes, used in this paper is presented. An experiment toward microblo data mining and analysis is shown in Section 4. Section 5 presents a conclusion.

\section{Related Work}

Bi et al. [17] proposed a methodology related to product/ service attributes for conducting IPA (importance-performance analysis) based on sentiment strengths of online reviews. And, more effective analysis results could be obtained by the proposed methodology with lower cost and shorter time. Lin et al. [18] proposed opinion-mined prototype based a tree-like retweeting structure to cope with the evolution of the microblog opinion. Sentiment was classified by an analysis method based microblog sentiment lexicon. Liu et al. [19] focused more on the subjective preference of the consumer and proposed an algorithm based on sentiment dictionaries to identify the positive, neutral, or negative sentiment orientation of online reviews. Feng et al. [20] proposed a novel hierarchial-structure LSTM model with context attention to do sentiment classification. Jiang et al. [21] used a six-dimensional vector, to compute sentiment of news event based on Weibo. In previous sentiment analysis work, short texts on social network are usually divided into texts with positive emotion and negative emotion. However, only positive and negative two polarity emotions are not enough to classify exactly the sentiment of the public in this study. Finally, data in this paper are manually classified into 4 emotion categories.

Deep neural network (DNN) has been used in many practical data analysis models $[22,23]$. Dos Santos and Gatti [24] denoted to do binary sentiment classification on short texts by a deep convolutional neural network (CNN) based on character- to sentence-level information. They achieved higher prediction accuracy on single sentence sentiment prediction. A new neural network approach proposed by Tang et al. [25], incorporating user-level and product-level information, worked for sentiment classification. Bi et al. [26] proposed an ensemble neural network-based model (ENNM), which could analyse complex relationships among factors obtained from online reviews, to measure the effects of customer sentiments toward different customer satisfaction dimensions (CSDs) on customer satisfaction. An effect-based Kano model (EKM) was proposed and used to classify the CSDs into different categories. Ibrahim and Yusoff [27] classified tweets into three categories by naïve Bayes classifier based on trainers' perception in order to improve the accuracy of text classification for sentiment analysis.

All in all, sentiment analysis on short texts based social networks is still popular topic in recent years. Considering Twitter and Facebook is not used commonly in China, this paper chooses social network Sina Weibo as text analysis platform. Referring to papers, this paper decides to compare different commonly-used methods, including CNN, DNN, and naïve Bayes to establish a more accurate festival classifier.

\section{Materials and Methods}

\subsection{Data Acquisition}

3.1.1. Platform Selection. In China, Weibo has gradually become an important part in people's daily life since Sina launched this service in 2009. Increasing number of people use this social platform to participate in the discussion of hot social topics. According to China Internet Network Information Centre (CNNIC), the user scale of Sina Weibo from 2014 to 2018 is obtained. As can be seen from the Figure 1, the user scale shows a continuous increase tendency with time going on, from 230 million to 351 million.

On one hand, Weibo achieves the integration of individuals and society through photos, short videos, retweets, comments and other functions. As a representative of Chinese new network media, Weibo provides platform for users to post actual emotions and opinions and make friends. On the other hand, the openness, interactivity, terminal scalability, operation simplicity, and big user scale make Sina Weibo quickly become one of the most important social media [5]. Therefore, a large amount of valuable research data is created by Sina Weibo. That is why Weibo is selected for this study.

3.1.2. Data Preprocessing. Python language is suitable to simulate functions such as microblog login and advanced search to write crawler program [28], so python crawler program is used to obtain data. The date and keywords of target festivals are set as index parameters. There are a lot of dirty data, including missing, duplicate, abnormal, and invalid ones in the original data returned by the program. In order to improve the quality of the data analysis in next phase, original data are necessary to be pretreated. In this 


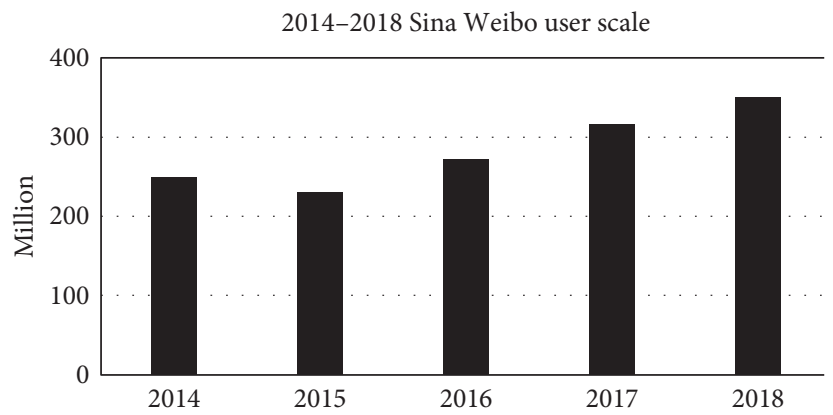

Figure 1: Sina Weibo user scale from 2014 to 2018.

paper, it is still necessary to control that there is only one microblog posted by a same user on the same day to guarantee the accuracy of analysing the public trend. Finally, text data are saved in CSV format after the processed data are read and labelled.

3.2. Text Augmentation by CHN-EDA. Because of the poor result of training small data set on the performance of text categorization, Wei and Zou [29] combined with previous work on automatic text data augment, testing a number of enhancement operation derived from the computer vision $[30,31]$ and then proposed EDA (easy data augmentation), that is obtaining a data growth model for English text through four processes: synonym replacement, random insertion, random swap and random deletion. Since the model is only applicable to English text, this paper uses CHN-EDA by referring to other Chinese models on the forum. By adjusting the weight of synonym substitution and synonym insertion, the required data are generated in proportion. An example of CHN-EDA is shown in Table 1, which illustrates four types of text augmentation. The bold parts are the variation points. This kind of enhancement operation is equivalent to generating new textual data with similar emotions. This method is not just replication of original text, it is also effective to solve the bias of the classification models.

3.3. Feature Extraction-Based TF-IDF. The word frequency of the top 1000 keywords of microblogs is obtained in the feature extraction steps, and the $1 * 1000$ dimension word frequency feature is extracted by the TF-IDF method. TFIDF reflects the importance of a word in the text, combining $\mathrm{TF}$ and IDF. The followings are the formulas of TF-IDF:

$$
\begin{array}{r}
i d f(t)=\log \left(\frac{n_{d}}{d f(t)+1}\right), \\
t f-i d f(t, d)=t f(t, d) * i d f(t),
\end{array}
$$

where idf(t) is term frequency-inverse document frequency, $n_{d}$ means the total number of documents, $d f(t)$ denotes the number of documents which contains word $t$, and $t f(t, d)$ is the frequency of the word $t$ in document $d$.
3.4. Deep Neural Network. DNN structure, shown in Figure 2, consists of input layer, hidden layer, and output layer. $1^{*} 1000$ dimension word frequency feature extracted by the TF-IDF is input, and then three fully-connected layers with 256,128 , and 64 neurons, respectively, compose the hidden layer. Neuron activation function is relu function, with Dropout used to increase the network structure to prevent training overfitting. Because of four categories of text classified in this paper, the output layer sets four neurons, using softmax function as the activation function.

3.5. Convolutional Neural Network. The CNN structure consists of input layer, convolution layer, fully-connected layer, and output layer, which is illustrated in Figure 3. The input layer is the $1^{*} 1000$ dimension word frequency feature extracted by TF-IDF method. In the first layer of convolution, one-dimensional convolution with $321^{*} 1000$ convolution kernels is used. Output of the first layer of convolution is $321^{*} 1$ features. The second layer convolution is a one-dimensional convolution with $321^{*} 32$ convolution kernels. The second layer convolution output are $321^{*} 1$ features. The fully connected layer contains two layers whose neurons numbers are 64 and 32, respectively, and the activation function is relu function. The Dropout function with a ratio of 0.5 is used. Finally, the output is divided into four categories by softmax function.

3.6. Naïve Bayes. Naïve Bayes has different classification thought with other classification algorithms in machine learning field. It relies on calculating probability distribution to achieve classification purpose instead of directly training the relation between output class tags and input eigenvectors.

$X\left(x_{1}, x_{2}, \ldots, x_{n}\right)$ is a input eigenvectors to be classified, and there are $m$ class tags $c_{1}, c_{2}, \ldots, c_{m}$ as output. $P\left(c_{1} \mid X\right), P\left(c_{2} \mid X\right), \ldots, P\left(c_{m} \mid X\right)$ is calculated to classify $X$, then the predicted category formula of $X$ is

$$
P\left(c_{k} \mid X\right)=\max \left\{P\left(c_{1} \mid X\right), P\left(c_{2} \mid X\right), \ldots, P\left(c_{m} \mid X\right)\right\} .
$$

According to the conditional independence assumption of naïve Bayes, each attribute is independent from each other. The conditional probability expression is as follows [32]: 
TABLe 1: Example of Chinese text augmentation by CHN-EDA.

\begin{tabular}{|c|c|}
\hline Operation & Sentence \\
\hline \multirow{2}{*}{ Original } & 清明找雨神 生无可恋的扫墓又塞车又热 \\
\hline & To find rain god on tomb-sweeping day. I had nothing left to live for with traffic jam and hot. \\
\hline \multirow{2}{*}{ Synonym replacement } & $\begin{array}{l}\text { 清明找雨神 生无可恋的扫墓又塞车又高热 } \\
\end{array}$ \\
\hline & To find rain god on tomb-sweeping day. I had nothing left to live for with traffic jam and heat. \\
\hline \multirow{2}{*}{ Random insertion } & 清明难免找雨神 生无可恋的扫墓又塞车又热 \\
\hline & To unavoidably find rain god on tomb-sweeping day. I had nothing left to live for with traffic jam and hot. \\
\hline \multirow{2}{*}{ Random swap } & 找清明雨神 生无可恋的扫墓又塞车又热 \\
\hline & To find tomb-sweeping day rain god. I had nothing left to live for with traffic jam and hot. \\
\hline \multirow{2}{*}{ Random deletion } & 找雨神 生无可恋的扫墓又塞车又热 \\
\hline & To find rain god. I had nothing left to live for with traffic jam and hot. \\
\hline
\end{tabular}

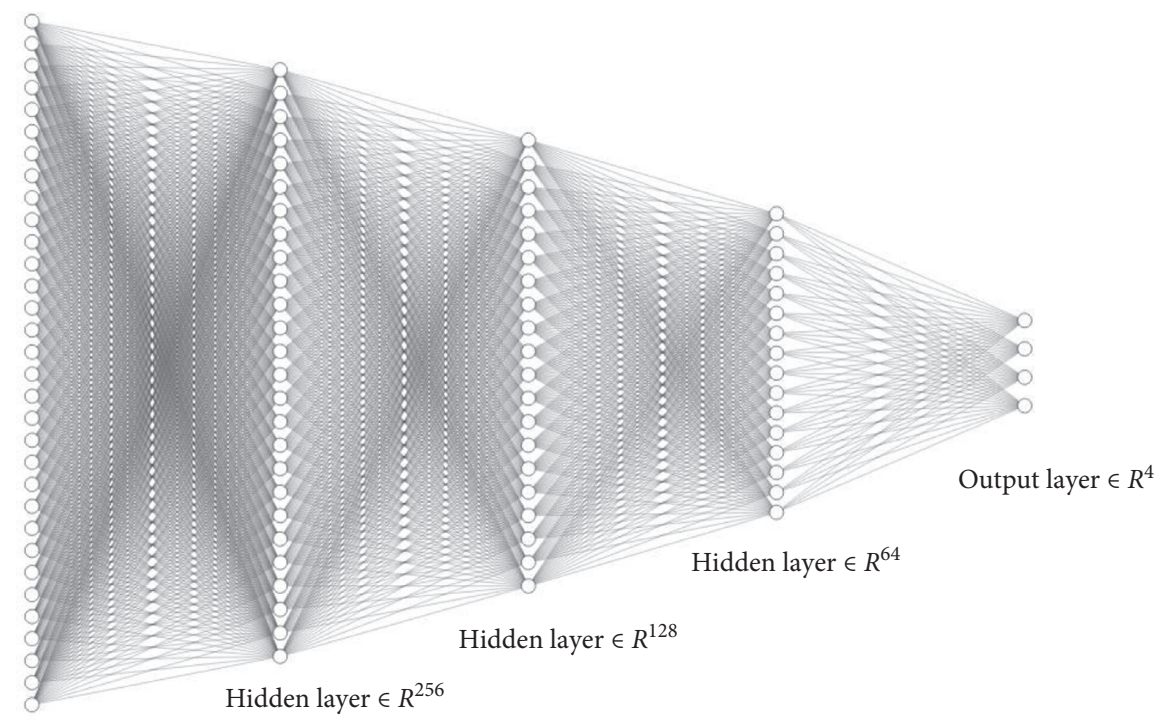

Input layer $\in R^{1000}$

FIGURE 2: Diagram of DNN structure.

$$
P(C \mid X)=\frac{P(X \mid C) P(C)}{P(X)}
$$

Take the comment "Happy Lantern Festival" as an example [28]:

$$
\begin{aligned}
& P(\text { Happy, Latern Festival|Joy })=P(\text { Happy|Joy }) * P(\text { Latern Festival|Joy }) \\
& P(\text { Joy } \mid \text { Happy, Latern Festival })=\frac{P(\text { Happy, Latern Festival } \mid \text { Joy }) * P(\text { Joy })}{P(\text { Happy, Latern Festival })} \\
& =\frac{P(\text { Happy } \mid \text { Joy }) * P(\text { Latern Festival } \mid \text { Joy }) * P(\text { Joy })}{P(\text { Happy, Latern Festival })} . \\
& P=\frac{a}{a+b} \\
& R=\frac{a}{a+c} \\
& F_{1}=\frac{2 \times P \times R}{P+R}
\end{aligned}
$$

\section{Experiment Results and Discussion}

4.1. Evaluation Indicators. The commonly used evaluation indicator [33], precision $(P)$, recall $(R)$, and $F_{1}$ score, is set to evaluate the proposed classifier in this paper. The parameters are shown in Table 2 and the formulas are calculated as 




Figure 3: Diagram of CNN structure.

TABLe 2: Parameters in evaluation.

\begin{tabular}{lcc}
\hline & Certain class label & Other class labels \\
\hline Recognized as certain class label by the classifier & $a$ & $b$ \\
Recognized as other class labels by the classifier & $c$ & $d$ \\
\hline
\end{tabular}

It is worth mentioning that, although these indicators are commonly used in the evaluation of binary classifier, it is also suitable to evaluate the effect of different class labels in such study [34]. To have a comprehensive consideration, accuracy (A) is also added into evaluation.

\subsection{Data Set}

4.2.1. Original Data from Crawler. Because this paper chooses traditional Chinese festivals as the objects of study, the crawler program is conducted to capture the data in the Dragon Boat Festival, Lantern Festival, Tomb-Sweeping Day, and Mid-Autumn Festival for five years (from 2014 to 2018). The program collects the data by setting the date and key words of the four festivals. In order to ensure the validity of data and the reliability of analysis, the size of data in different festivals needs to be in balance. In total, there are 29574 microblogs crawled from Sina Weibo as experimental text data. An example of the related data obtained by crawlers is shown in Table 3 . The collected dimensions contain date, microblog ID, username, user title (including Weibo membership, Weibo personal authentication, Weibo official authentication, Weibo talent, and none), microblogs, number of retweets, and number of comments.

4.2.2. Manual Label. The traditional sentiment classifier is to perform the binary classification of the texts, that is, the emotion of a text is positive or negative. However, this kind of classifier is not enough to know exactly the sentiment of the public in some situations. In this paper, 29574 microblogs are manually classified into 4 emotion categories in which emotions are divided as joy, angry, bored, and sad. Numbers of data with 4 different labels are shown in Table 4, including 26059 microblogs with joy emotion, 233 ones with angry emotion, 121 bored ones, and $3161 \mathrm{sad}$ ones. Information in Table 5 illustrates examples of data with manual labels.

4.2.3. Process of $C H N-E D A$. Based on the results of the manual label of the data, there is a serious imbalance in the data. For example, there are 26059 microblogs belonging to joy category, but only 3161 ones belonging to sad and 122 belonging to bored. Such question above can lead to problems like overfitting, which then results in a decrease in the accuracy of the classifier. It can be seen from Table 6 that only joy category has a greater than $90 \%$ precision. Precision, recall, and $F_{1}$ score of data with angry label are just $19 \%, 15 \%$, and $17 \%$, respectively. The $F_{1}$ score of $s a d$ label is $51 \%$, which is a little better than bored label whose $F_{1}$ score is just $11 \%$. According to existing literatures [34], some relative solution, such as resampling technique which is just duplicating texts, cannot guarantee the quality of the data. The effect of backtranslation technique [35] depends on the quality of translation. Syntax, sentence extension, and contraction produce sentences with similar structure to the original sentence, but this operation is not easy to achieve and may result in the loss of semantic information. Finally, the CHN-EDA-based on EDA technique [29] is used to address the imbalance issue after comparing some currently applied methods' advantage and disadvantages.

Remarkable results are shown in Table 7 after using CHN-EDA text augmentation. For joy label, the improvement of classification effect is not obvious after CHN-EDA processing. This is because that too much joy-labelled data in the original data set result in $88 \%$ data with joy label in the test and training sets, which leads to the bias of the model. In 
TABLE 3: Example of the Weibo information obtained by the crawler.

\begin{tabular}{lc}
\hline Date & $2018-3-2$ \\
\hline Microblog ID & 4213002779060242 \\
Username & 好姑娘来自北方 (good girl from northern) \\
User title & 微博会员 (Weibo membership) \\
Microblogs & 好友早安 元宵节快乐 (good morning friends happy lantern festival) \\
Number of retweets & 7004 \\
Number of comments & 509 \\
\hline
\end{tabular}

TABle 4: Numbers of data with different labels after manual labelled.

\begin{tabular}{lcccr}
\hline Dataset & Joy & Angry & Bored & \\
\hline 29574 & 26059 & 233 & 121 & 3161 \\
\hline
\end{tabular}

TABLE 5: Examples of data with different labels.

\begin{tabular}{lccc}
\hline Joy & Angry & Bored & Sad \\
$\begin{array}{l}\text { 早上好, 端午节快 } \\
\begin{array}{l}\text { 乐 (good morning, } \\
\text { happy dragon boat } \\
\text { festival ) }\end{array}\end{array}$ & $\begin{array}{c}\text { 好好的元宵节 遛个狗也能被气 } \\
\text { (walking a dog can be angry to }\end{array}$ & $\begin{array}{c}\text { 最讨厌的是过节! 讨厌鞭炮声 } \\
\text { (hate festivals mostly! hate the } \\
\text { sound of firecrackers.) }\end{array}$ & $\begin{array}{c}\text { 悲剧的中秋节 (a tragic mid- } \\
\text { autumn festival) }\end{array}$ \\
$\begin{array}{l}\text { 中秋快乐, 一家团圆 } \\
\text { (happy mid-autumn } \\
\text { festival, a family reunion.) }\end{array}$ & $\begin{array}{c}\text { 今年的端午节礼物, 火车晚点三 } \\
\text { 个时!! (dragon boat festival gift } \\
\text { this year, the train delays three } \\
\text { hours!!) }\end{array}$ & $\begin{array}{c}\text { 中秋节 坚决不吃月饼 恶心 } \\
\text { (mooncakes are definitely not eaten } \\
\text { on mid-autumn festival. Disgusting) }\end{array}$ & $\begin{array}{c}\text { 2016年的清明节, 心痛的无法呼 } \\
\text { too much heartache to breath.......) }\end{array}$ \\
\hline
\end{tabular}

TABLE 6: Original emotion classification results.

\begin{tabular}{lccr}
\hline Emotion category & Precision & Recall & \\
\hline Joy & 0.93 & 0.95 & 0.94 \\
Angry & 0.19 & 0.15 & 0.17 \\
Bored & 0.10 & 0.12 & 0.11 \\
Sad & 0.54 & 0.48 & 0.51 \\
\hline
\end{tabular}

Table 7: Emotion classification results after using CHN-EDA.

\begin{tabular}{lccc}
\hline Emotion category & Precision & Recall & $F_{1}$ \\
\hline Joy & 0.95 & 0.91 & 0.93 \\
Angry & 0.93 & 0.83 & 0.88 \\
Bored & 0.92 & 0.87 & 0.90 \\
Sad & 0.79 & 0.90 & 0.84 \\
\hline
\end{tabular}

other words, if a new data is predicted, the model is more inclined to classify it as a joy label. It is true that there is indeed more joy label data, so it will probably be shown that the classification accuracy of the label is very high, but it does not represent the actual prediction accuracy. Among the remaining three labels, sad increases the label precision, recall rate, and $F_{1}$ by $25 \%, 42 \%$, and $33 \%$, although the number is not as much as that of angry and bored whose rates rise by nearly $80 \%$. The reason is that sad label data is the second most data label in original data set, so its prediction is also influenced much due to the bias impression of the model. Overall, what can be learned from the results is that it is necessary for the original data set to be processed by CHN-EDA.

\subsection{Festival Classifier Establishment}

4.3.1. Sentiment Analysis Method Comparison. Traditional sentiment analysis methods, including CNN, $\mathrm{DNN}$, and naïve Bayes, are compared to establish more efficient sentiment classifier. The accuracy and average performance (the average accuracy of different labels classification) of different classifiers are tested before and after using CHN-EDA data augmentation. As can be seen from Table 8 , on one hand, better results can be obtained by all the algorithms after the text augmentation. Under the CNN method, the overall classification accuracy of the classifier is improved by nearly 3\%. The classifier accuracy increases from $89.61 \%$ to $93.76 \%$ with the DNN method. Although the 
TABLe 8: Performance under different models.

\begin{tabular}{lcc}
\hline Model & Accuracy & Average performance \\
\hline CNN & 0.8797 & 0.9398 \\
+CHN-EDA & 0.9073 & 0.9536 \\
DNN & 0.8961 & 0.9480 \\
+CHN-EDA & 0.9376 & 0.9688 \\
Naïve Bayes & 0.4645 & 0.9404 \\
+CHN-EDA & 0.5247 & 0.8352 \\
\hline
\end{tabular}

classification effect of naïve Bayes is not ideal, it still could be seen that the classification accuracy is significantly improved after EDA data augmentation. The above experimental results also prove the necessity and effectiveness of using CHN-EDA in this festival classifier. On the other hand, the traditional naïve Bayes classifier shows lower accuracy and the average performance is also lower than that of CNN and DNN classifiers, which indicates that the classification of different labels is not accurate enough, so naïve Bayes is not taken into account in the following. Combined with the selection of the optimizer, CNN and DNN methods will be compared further later.

4.3.2. Sentiment Analysis Algorithm Complexity Comparison. The time complexity of neural network algorithm is related to many variables such as epochs, dataset size, number of layers, and convolution kernel size and requires intralayer multiplication and interlayer accumulation, making the calculation more complex. So, it is not easy to compare the neural network complexity directly because it is hard to use a simple formula or mathematical expression to represent it accurately. However, since small changes in each variable can cause changes in the calculation time, running time of the algorithms can reflect the algorithm complexity to a certain extent. Thus, the algorithm complexity is compared in this paper by comparing the running time of the algorithms.

On the basis of comparing the accuracy of $\mathrm{CNN}$ and DNN algorithm, this section verifies the running rate of the two algorithms. To compare the algorithm complexity, the models use python 3 to execute the tensorflow framework on a win 10 system computer with an $17-8700 \mathrm{k} 3.7 \mathrm{GHz}$ Intel core CPU, 32 GB of memory, and GTX2080 8 GB GPU. The running time of $\mathrm{CNN}$ and $\mathrm{DNN}$ algorithm is tested under the same conditions. The experiment runs for a total of 10 times to reduce the uncertainty during operation, and the results of the 10 times are averaged as the final result, as shown in Table 9. The running time of the algorithm is measured in seconds.

By comparing the running time of the two algorithms, it can be seen that the average running time of CNN algorithm with 10 tests is 37.803 seconds, while the average running time of DNN algorithm is 33.187 seconds. DNN algorithm is superior to CNN algorithm in time performance with obvious advantages.
4.3.3. Optimizer Selection. In the process of constructing the optimal classifier, the optimizer with better matching effect needs to be selected. Although some experimental results show that many optimizers have good optimization effect, it is hard to find the optimal one objectively. Combined with the specific problems studied in this paper, the more suitable optimizer and the optimal matching parameters are confirmed through relevant experiments.

Firstly, six optimizers that are widely used in the field of machine learning, namely, Adagrad, Adam, Nadam, RMsprop, and SGD are selected. With the classification accuracy of the four labels as the evaluation index of this section, the effect of the classifier is compared under DNN and CNN methods, respectively. It can be seen from Figures 4 and 5 that the SGD optimizer has obvious differences and disadvantages from the other four optimizations. The classification accuracy of joy label with SGD optimizer is just $67 \%$, which is much lower than other optimizers with more than $90 \%$ accuracy. And, models with SGD optimizer show $75 \%$ accuracy in sad label while other optimizers have accuracy about $93 \%$. So, the SGD optimizer is not used as the research object in the following experiments. The classification accuracy of labels cannot clearly reflect the significant difference in the optimization effect of the remaining optimizer. Therefore, $F_{1}$ score is then used as the baseline for optimizer comparison, because this indicator considers both precision rate and recall rate, so as to get a more accurate and objective comparison conclusion.

Comparison between Figures 6 and 7 shows $F_{1}$ scores of most labels' classification under the DNN method are about $95 \%$ which is higher than that of labels under the CNN method. The scores in angry label under $\mathrm{CNN}$ are lower than $90 \%$. The indicators are even less than $85 \%$ in sad label. Above all, $F_{1}$ score comparison results show that firstly, the overall operation effect of DNN is higher than that of CNN. Secondly, in terms of optimization ability of the optimizer, Adagrad is more suitable for the optimization algorithm of this classifier.

4.3.4. Learning Rate of the Optimizer. This section determines the final learning rate of the optimizer through experiments. The results in Figure 8 show, when the learning rate is 0.05 , the accuracy of DNN sentiment analysis classifier reaches the highest point, that is, $94 \%$. From Figure 8, it can also be found that, in this 
TABLE 9: Comparison of algorithms in running time.

\begin{tabular}{|c|c|c|c|c|c|}
\hline \multirow{2}{*}{ Number of runs } & \multicolumn{2}{|c|}{ Algorithm run time } & \multirow{2}{*}{ Number of runs } & \multicolumn{2}{|c|}{ Algorithm run time } \\
\hline & $\mathrm{CNN}$ & DNN & & $\mathrm{CNN}$ & DNN \\
\hline 1 & 37.25 & 33.50 & 6 & 38.35 & 33.40 \\
\hline 2 & 36.92 & 33.95 & 7 & 37.67 & 33.04 \\
\hline 3 & 37.17 & 33.51 & 8 & 38.46 & 32.69 \\
\hline 4 & 37.08 & 33.49 & 9 & 38.69 & 32.16 \\
\hline 5 & 37.91 & 33.46 & 10 & 38.53 & 32.67 \\
\hline Average run time & 37.803 & 33.187 & & & \\
\hline
\end{tabular}

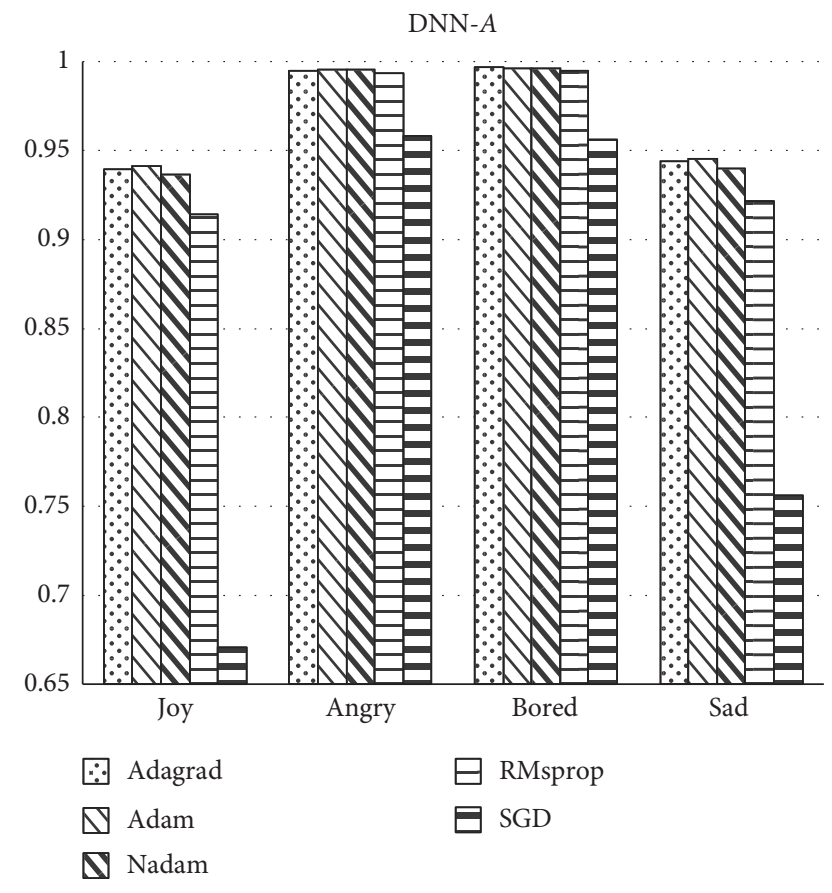

FIgURE 4: Accuracy of labels classification under the DNN method.

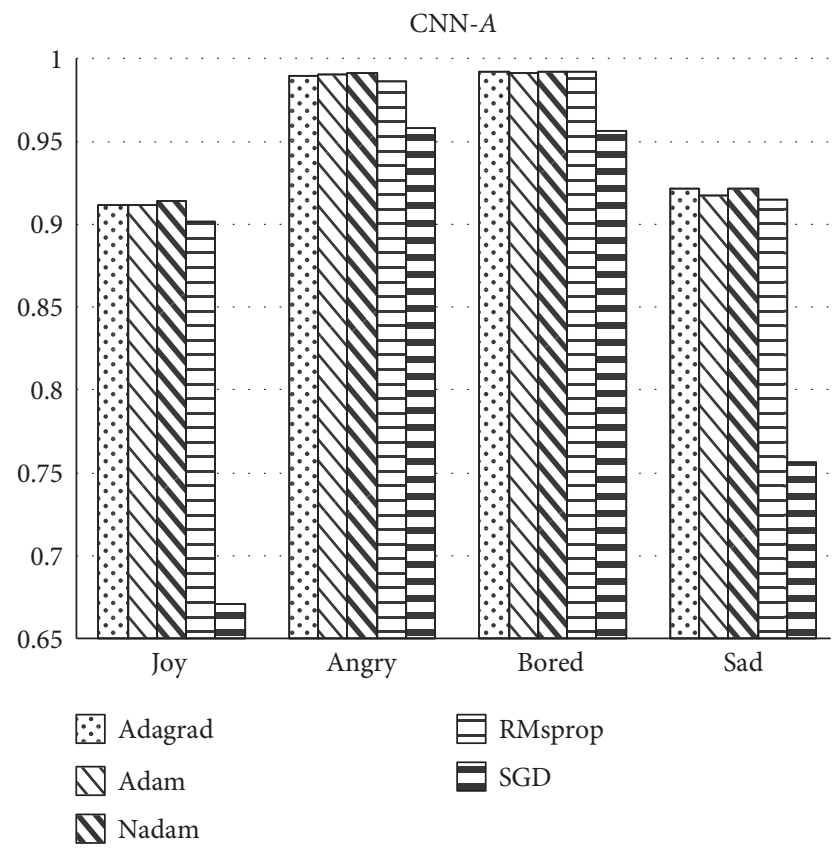

Figure 5: Accuracy of labels classification under the CNN method.

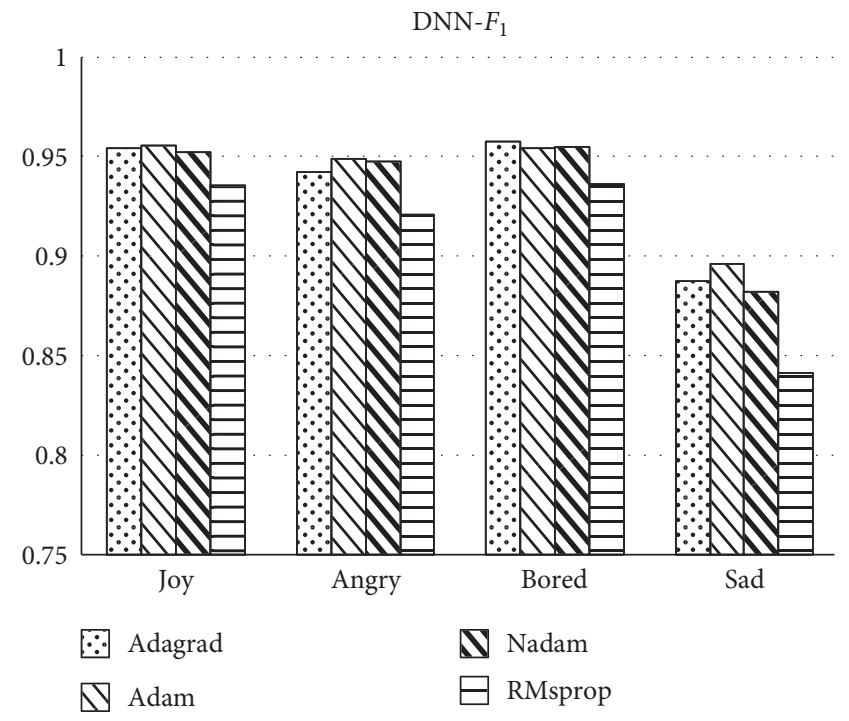

FIgURE 6: $F_{1}$ score of labels classification under the DNN method.

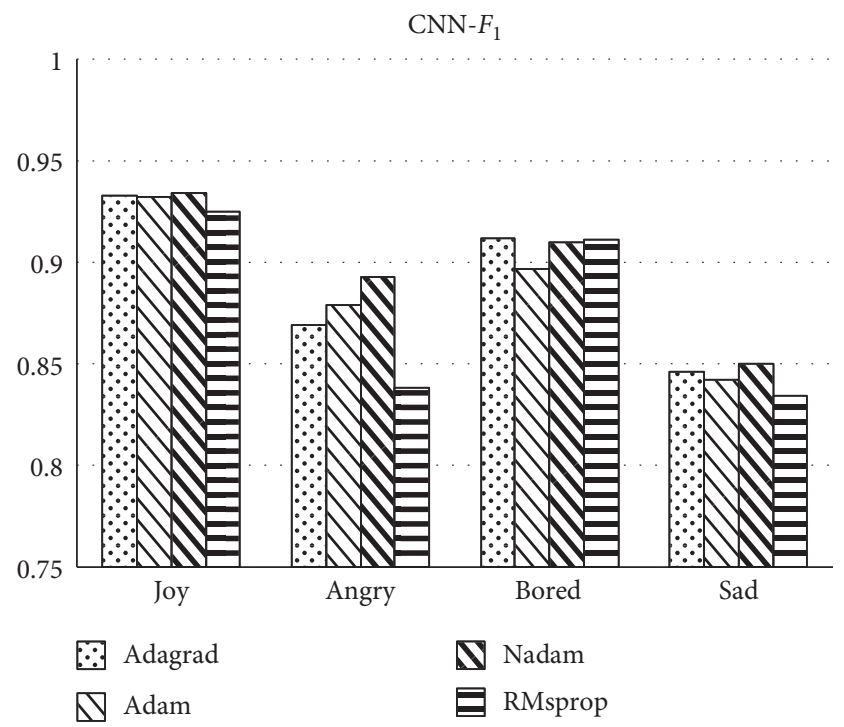

FIgURE 7: $F_{1}$ score of labels classification under the CNN method.

environment, the accuracy of DNN method is always higher than that of CNN. Combined with the above experiments, it can be concluded that the DNN algorithm is more suitable for this study. DNN is finally identified as 


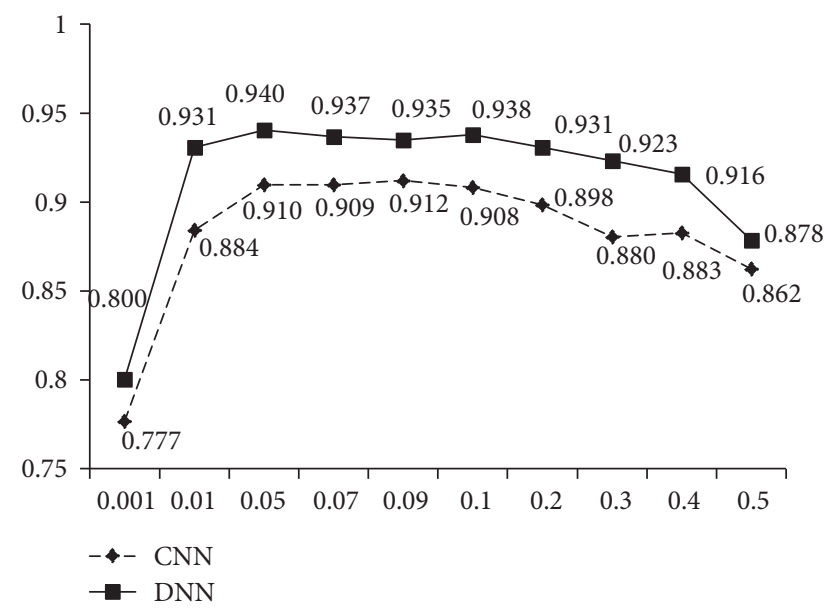

FIgURe 8: Accuracy of classifiers with different learning rates.

the festival sentiment classifier and Adagrad as the matching optimizer.

\section{Conclusions}

Through experiments, after comparing different text analysis methods, CNN, DNN, and naïve Bayes, the method and parameter values for festival classifier can be determined finally. Experiment results illustrate DNN as sentiment analysis model and the Adagrad optimizer with a learning rate of 0.05 are suitable to build such a classifier.

The major contribution of this paper is that this paper combines the festival data and sentiment analysis models, establishing a Chinese festival classifier under the traditional festivals background, which can obtain public's festival sentiment effectively. Moreover, CHN-EDA is applied for solving the bias of the model caused by sentiment microblog imbalance. Through changing weight of synonym substitution and synonym insertion, a suitable dataset is generated in proportion automatically.

In the future research, more analytical methods can be compared to explore a more efficient classification model. And, such thought can be applied to the business decision and festival sentiment trend analysis, in order to play its greater practical value.

\section{Data Availability}

Original social text data are obtained by Python crawler programming, which is operated by setting the date and keyword as index parameters of the microblogs. All the data used in this paper are collected from the Sina Weibo (https:// weibo.com), one of the most popular social networks in China.

\section{Conflicts of Interest}

The authors declare that they have no conflicts of interest.

\section{Acknowledgments}

This research was funded by the Northeastern University Industry-University-Research Strategic Cooperation Project (code: 71971050).

\section{References}

[1] R. C. J. Chia, S. Y. Lim, P. K. Ong, and S. F. Teh, "Pre and post Chinese new year holiday effects: evidence from Hong Kong stock market," The Singapore Economic Review, vol. 60, no. 4, p. 1550023, 2015.

[2] T. Yuan and R. Gupta, "Chinese lunar new year effect in asian stock markets, 1999-2012," The Quarterly Review of Economics and Finance, vol. 54, no. 4, pp. 529-537, 2014.

[3] L. Yue, W. Chen, X. Li, W. Zuo, and M. Yin, "A survey of sentiment analysis in social media," Knowledge and Information Systems, vol. 60, no. 2, pp. 617-663, 2019.

[4] J. Aguilar and O. Terán, "Social media and free knowledge: case study-public opinion formation," in Politics and Social Activism: Concepts, Methodologies, Tools, and Applications, pp. 433-466, IGI Global, Hershey, PA, USA, 2016.

[5] L. Li, Y. Wu, Y. Zhang, and T. Zhao, "Time + user dual attention based sentiment prediction for multiple social network texts with time series," IEEE Access, vol. 7, pp. 17644-17653, 2019.

[6] S. G. S. Fernando, "Empirical analysis of data mining techniques for social network websites," Compusoft, vol. 3, no. 2, p. $582,2014$.

[7] R. Xia, J. Jiang, and H. He, "Distantly supervised lifelong learning for large-scale social media sentiment analysis," IEEE Transactions on Affective Computing, vol. 8, no. 4, pp. 480491, 2017.

[8] V. N. Patodkar and I. R. Sheikh, "Twitter as a corpus for sentiment analysis and opinion mining," International Journal of Advanced Research in Computer and Communication Engineering, vol. 5, no. 12, pp. 320-322, 2016.

[9] A. Viahal and S. S. Sonawane, "Sentiment analysis of twitter data: a survey of techniques," International Journal of Computer Applications, vol. 139, no. 11, pp. 5-15, 2016.

[10] S. T. Russell and S. T. Horn, Sexual Orientation, Gender Identity, and Schooling: The Nexus of Research, Practice, and Policy, Springer, Berlin, Germany, 2017.

[11] D. Deng, L. Jing, J. Yu, and S. Sun, "Sparse self-attention LSTM for sentiment lexicon construction," IEEE/ACM Transactions on Audio, Speech, and Language Processing, vol. 27, no. 11, pp. 1777-1790, 2019.

[12] J. Wu, K. Lu, S. Su, and S. Wang, "Chinese micro-blog sentiment analysis based on multiple sentiment dictionaries and semantic rule sets," IEEE Access, vol. 7, pp. 183924183939, 2019.

[13] H. Deng, J. Han, H. Li, H. Ji, H. Wang, and Y. Lu, "Exploring and inferring user-user pseudo-friendship for sentiment analysis with heterogeneous networks," Statistical Analysis and Data Mining: The ASA Data Science Journal, vol. 7, no. 4, pp. 308-321, 2014.

[14] K. Ravi and V. Ravi, "A survey on opinion mining and sentiment analysis: tasks, approaches and applications," Knowledge-Based Systems, vol. 89, no. 89, pp. 14-46, 2015.

[15] D. Zhang, H. Xu, Z. Su, and Y. Xu, "Chinese comments sentiment classification based on word2vec and SVMperf," Expert Systems with Applications, vol. 42, no. 4, pp. 1857-1863, 2015.

[16] Z. Hao, R. Cai, Y. Yang, W. Wen, and L. Liang, "A dynamic conditional random field based framework for sentence-level sentiment analysis of Chinese microblog," in Proceedings of the 2017 IEEE International Conference on Computational Science and Engineering (CSE) and IEEE International Conference on Embedded and Ubiquitous Computing (EUC), pp. 135-142, Guangzhou, China, July 2017. 
[17] J.-W. Bi, Y. Liu, Z.-P. Fan, and J. Zhang, "Wisdom of crowds: conducting importance-performance analysis (IPA) through online reviews," Tourism Management, vol. 70, pp. 460-478, 2019.

[18] L. Lin, L. Jianxin, R. Zhang, W. Yu, and C. Sun, "Opinion mining and sentiment analysis in social networks: a retweeting structure-aware approach," in Proceedings of the 2014 IEEE/ACM 7th International Conference on Utility and Cloud Computing, London, UK, December 2014.

[19] Y. Liu, J.-W. Bi, and Z.-P. Fan, "Ranking products through online reviews: a method based on sentiment analysis technique and intuitionistic fuzzy set theory," Information Fusion, vol. 36, pp. 149-161, 2017.

[20] S. Feng, Y. Wang, L. Liu, D. Wang, and G. Yu, "Attention based hierarchical LSTM network for context-aware microblog sentiment classification," World Wide Web, vol. 22, no. 1, pp. 59-81, 2019.

[21] D. Jiang, X. Luo, J. Xuan, and Z. Xu, "Sentiment computing for the news event based on the social media big data," IEEE Access, vol. 5, pp. 2373-2382, 2017.

[22] X. W. Chen and X. Lin, "Big data deep learning: challenges and perspectives," IEEE Access, vol. 2, pp. 514-525, 2014.

[23] A. Severyn and A. Moschitti, "UNITN: training deep convolutional neural network for twitter sentiment classification," in Proceedings of the 9th international workshop on semantic evaluation (SemEval 2015), pp. 464-469, Denver, CO, USA, June 2015.

[24] C. Dos Santos and M. Gatti, "Deep convolutional neural networks for sentiment analysis of short texts," in Proceedings of the COLING 2014, the 25th International Conference on Computational Linguistics, pp. 69-78, Dublin, Ireland, August 2014.

[25] D. Tang, B. Qin, and T. Liu, "Learning semantic representations of users and products for document level sentiment classification," in Proceedings of the 53rd Annual Meeting of the Association for Computational Linguistics and the 7th International Joint Conference on Natural Language Processing (Volume 1: Long Papers), pp. 1014-1023, Beijing, China, July 2015.

[26] J.-W. Bi, Y. Liu, Z.-P. Fan, and E. Cambria, "Modelling customer satisfaction from online reviews using ensemble neural network and effect-based Kano model," International Journal of Production Research, vol. 57, no. 22, pp. 7068-7088, 2019.

[27] M. N. M. Ibrahim and M. Z. M. Yusoff, "Twitter sentiment classification using Naive Bayes based on trainer perception," in Proceedings of the 2015 IEEE Conference on e-Learning, e-Management and e-Services (IC3e), Melaka, Malaysia, August 2015 .

[28] Z. J. Ren, P. Zhang, S. C. Li, Y. X. Lan, X. Xia, and Y. C. Cui, "Analysis of emotion evolution of emergencies based on Weibo data mining: taking " 8.12 Accident in Tianjin" as an example," Journal of Intelligence, vol. 38, no. 2, pp. 140-148, 2019.

[29] J. Wei and K. Zou, "EDA: easy data augmentation techniques for boosting performance on text classification tasks," in Proceedings of the 2019 Conference on Empirical Methods in Natural Language Processing and the 9th International Joint Conference on Natural Language Processing (EMNLPIJCNLP), pp. 6383-6389, Hong Kong, China, November 2019.

[30] C. Szegedy, W. Liu, Y. Jia et al., "Going deeper with convolutions," in Proceedings of the IEEE conference on computer vision and pattern recognition, pp. 1-9, Boston, MA, USA, July 2015.
[31] A. Krizhevsky, I. Sutskever, and G. E. Hinton, "Imagenet classification with deep convolutional neural networks," Communications of the ACM, vol. 60, no. 6, pp. 84-90, 2017.

[32] W. K. Li, P. Y. Liu, Z. F. Zhu, and W. F. Liu, "Sentence classification model based on convolution neural. network and Bayesian classifier," Application Research of Computers, vol. 37, no. 2, pp. 333-336+341, 2020.

[33] G. XU, Y. Meng, X. Qiu, Z. Yu, Z. Yu, and X. Wu, "Sentiment analysis of comment texts based on BiLSTM," IEEE Access, vol. 7, pp. 51522-51532, 2019.

[34] J. Yan, K. Wang, Y. Liu et al., "Mining social lending motivations for loan project recommendations," Expert Systems with Applications, vol. 111, pp. 100-106, 2018.

[35] A. W. Yu, D. Dohan, and Q. Le, "Fast and accurate reading comprehension by combining self-attention and convolution," in Proceedings of the International Conference on Learning Representations, Vancouver, Canada, May 2018. 